\title{
"PESQUISADOCS": ELEMENTOS PARA A CARTOGRAFIA DA COMUNIDADE BRASILEIRA DE PESQUISADORES EM ORGANIZAÇÃO DO CONHECIMENTO
}

\author{
"PESQUISADOCS": ELEMENTS FOR THE \\ CARTOGRAPHY OF THE KNOWLEDGE ORGANIZATION \\ BRAZILIAN COMMUNITY OF RESEARCHERS
}

\author{
Walter Moreira \\ Fernanda Carolina Pegoraro Novaes ${ }^{b}$ \\ Amanda Mendes da Silvac
}

\begin{abstract}
RESUMO
Objetivo: Considerando-se o tamanho e a dispersão geográfica da comunidade de pesquisadores que estuda a temática da organização do conhecimento, objetiva-se identificar, descrever e mapear, com o recurso de coordenadas geográficas, os centros de referência e os pesquisadores envolvidos com a pesquisa brasileira em organização do conhecimento. Metodologia: Os referidos pesquisadores, vinculados a programas de pós-graduação no Brasil, foram identificados por meio dos respectivos currículos Lattes e, adicionalmente, pela coleta de informações junto às páginas web dos programas em que atuam. Utilizou-se como recurso tecnológico para a coleta de dados e para a construção e disponibilização da informação cartográfica o Google Maps. Resultados: Nesta primeira aproximação, o mapa produzido identifica e cataloga um conjunto de 24 docentes distribuídos em 9 instituições cobrindo todo o território brasileiro. Conclusões: O mapa apresentado possibilita localizar e identificar rapidamente endereços, contatos e outras informações breves de pesquisadores cujos temas centrais se relacionem à organização do conhecimento, facilitando eventuais contatos, inclusive com apresentação de coordenadas geográficas, o que pode ser utilizado para a exportação dos dados cadastrados para outras bases ou softwares de gestão da informação cartográfica mais robustos.
\end{abstract}

Descritores: Organização do conhecimento. Comunidade de pesquisadores. Cartografia.

a Doutor em Ciência da Informação pela Universidade de São Paulo (USP). Docente do Departamento de Ciência da Informação da Universidade Estadual Paulista (UNESP). E-mail: walter.moreira@unesp.br.

b Doutoranda do Programa de Pós-Graduação em Ciência da Informação da Universidade Estadual Paulista (UNESP). E-mail: fernanda.pegoraro@unesp.br.

c Mestranda do Programa de Pós-Graduação em Ciência da Informação da Universidade Estadual Paulista (UNESP). E-mail: amanda.m.silva@unesp.br. 


\section{INTRODUÇÃO}

Quando se faz referência à ideia de comunidade de pesquisadores, temse em mente que o elemento que os agrega é o próprio interesse dos membros, isto é, entre um pesquisador que atua num programa de pós-graduação em ciência da informação no interior do estado de São Paulo, por exemplo, e um outro pesquisador que exerce atividades semelhantes no sul da Itália, haveria conexões inerentes formadas pelos interesses de pesquisa comuns a ambos. Essa noção de comunidade, contudo, apesar de válida, é semanticamente fraca, pois o conceito de comunidade requer a característica de compartilhamento efetivo, ou seja, trata-se de um conjunto de pessoas unidas por interesses comuns, que mantêm contatos entre si de forma síncrona ou assíncrona, presencial ou mediada por tecnologias, de modo que possam trocar experiências, ideias, projetos e outros.

O conceito de colégio virtual, viabilizado pelas tecnologias digitais, facilita a identificação, a localização e a intercomunicação entre os membros de uma coletividade, permitindo sobrepor seus interesses de pesquisa às barreiras geográficas e até mesmo linguísticas, em alguns casos. Essa rede virtual de relacionamentos virtual define-se, na prática, como uma rede real, com resultados efetivos, pois se trata de um grupo de pesquisadores irmanados por interesses comuns e que trocam informações via correio eletrônico, listas de discussões, redes sociais ou outros meios digitais de comunicação, promovendo a socialização do conhecimento entre a comunidade científica (MOREIRA, 2005).

Há, entretanto, questões a respeito dos efeitos práticos dos colégios virtuais que ainda requerem investigação, tais como, por exemplo, a identificação do perfil dos pesquisadores que atuam como provedores de informação nestes grupos, a natureza dessa informação, o mapeamento dos interesses de pesquisa e das redes locais, regionais, nacionais ou internacionais que se constroem para esses fins. Para aproximar uma resposta a questões como essas e outras que poderiam amparar o desenvolvimento de políticas de informação e de desenvolvimento de pesquisas é necessário, antes, dimensionar e descrever 
corretamente a comunidade de pesquisadores envolvida.

Desse modo, tomando-se a organização do conhecimento como objeto de estudo, a questão geral que orienta este trabalho diz respeito ao mapeamento da comunidade científica de pesquisadores que atuam em território brasileiro cujas temáticas de pesquisa se relacionem com a organização e a representação do conhecimento.

Como primeiro passo, apresenta-se, portanto, neste trabalho, o resultado de um projeto-piloto desenvolvido para a identificação e a criação de uma base de dados da comunidade brasileira de pesquisadores da organização e representação do conhecimento, com recursos da cartografia e das tecnologias digitais de representação da informação cartográfica.

\section{CAMPO DA ORGANIZAÇÃO DO CONHECIMENTO}

Considerando-se o seu percurso histórico, a organização do conhecimento pode ser compreendida a partir de três momentos destacados: a) como "arte", isto é, de forma mais intuitiva; b) como técnica, a partir da segunda metade século XIX, preocupada com o desenvolvimento de um conjunto de regras para a sistematização de conceitos; c) como metodologia, a partir da segunda metade século XX, em busca de uma base científica para o conjunto de procedimentos (GUIMARÃES, 2003).

Atualmente, a organização do conhecimento é um campo consolidado de pesquisas teóricas e aplicadas sobre o fenômeno do conhecimento, contemplando, de modo não exclusivo, os aspectos epistemológicos relativos aos domínios, a interdisciplinaridade do conhecimento e as questões culturais e éticas relativas à sua organização e representação, bem como condições necessárias à recuperação da informação.

Apesar de ser um processo individualizado, o conhecimento não é apenas individual, pois é também social, uma vez que todo conhecimento diz respeito de modo direto a culturas, valores e princípios. Assim, quando fatores sociais interferem na visão de mundo do ser humano, o conhecimento será subjetivo (inerente ao sujeito) e quando essa pessoa interage com um novo conhecimento, isso revelará sua faceta social. $O$ objeto de estudo da organização do 
conhecimento, como afirma Barité (2001, p. 42), é o conhecimento socializado. A primeira das dez premissas básicas para a compreensão da organização do conhecimento, apresentadas por esse autor, assevera que o conhecimento é "um produto social, uma necessidade social e um dínamo social". Coloca-se, desse modo, a socialização do conhecimento como elemento indispensável que se atrela tanto ao efeito prático resultante das ações de organização do conhecimento quanto às práticas de pesquisa da comunidade de pesquisadores desse campo.

Um marco na construção da comunidade de pesquisadores e na institucionalização da organização do conhecimento foi a fundação, em 1989, da International Society for Knowledge Organization (ISKO), reunindo pesquisadores da ciência da informação, da filosofia e da linguística, entre outras áreas, com o propósito de obter avanços teóricos e metodológicos relativos ao campo. Atualmente, a ISKO, além de colaborar com a construção do conhecimento científico de maneira interdisciplinar, é responsável pela promoção do diálogo entre pesquisadores do mundo todo. Um dos instrumentos que utiliza para isso é a promoção de congressos científicos internacionais, nacionais e regionais, sendo estes últimos denominados como capítulos (GUIMARÃES, 2017).

O braço da ISKO no Brasil, chamado ISKO Brasil, organiza e promove a cada dois anos o "Congresso Brasileiro em Organização e Representação do Conhecimento", cuja edição mais recente, das cinco já realizadas, ocorreu em 2019, na cidade de Belém - PA. A efetividade da presença e das ações da ISKO no Brasil corrobora a importância dos pesquisadores brasileiros também no cenário internacional.

Além do capítulo ISKO, o Brasil conta com um segundo evento igualmente importante para a comunidade de pesquisadores em organização do conhecimento: o Encontro Nacional de Pesquisa em Ciência da Informação (ENANCIB), promovido anualmente pela Associação Nacional de Pesquisa e Pós-Graduação em Ciência da Informação (ANCIB), com vinte edições realizadas até o ano de 2020. Nesse evento, destacam-se, para os interesses da comunidade de pesquisadores em tela, os trabalhos apresentados no grupo 
de trabalho "GT2 - Organização e representação do conhecimento".

Considerando-se, portanto, a extensão da comunidade de pesquisadores em organização do conhecimento no Brasil, a necessidade de promover interlocuções e parcerias, bem como, adicionalmente, a vasta extensão territorial do Brasil como um fator crítico para as aproximações necessárias, apresentamse como objetivos para este trabalho: a) identificar os centros de referência em discussões sobre organização do conhecimento no Brasil; b) localizar e identificar os pesquisadores brasileiros cujos temas de pesquisa se relacionem à organização do conhecimento; c) criar coordenadas geográficas para a localização dos centros de referência identificados e seus pesquisadores, com recursos de cartografia e de tecnologias digitais.

A pesquisa justifica-se pela necessidade de uma base de dados georreferenciada que facilite a identificação, os contatos e a integração entre membros ou interessados na comunidade de pesquisadores brasileiros envolvidos com a organização do conhecimento, além de colaborar adicionalmente para a identificação dos pares e a construção da memória da organização do conhecimento no Brasil.

\section{A CARTOGRAFIA E O CIBERESPAÇO}

Segundo o Dicionário Cartográfico publicado pelo IBGE (OLIVEIRA, 1993), a cartografia refere-se a um conjunto teórico-aplicado que utiliza recursos científicos, técnicos e artísticos para elaborar e utilizar, com base em observações diretas ou pesquisas documentais, expressões gráficas ou representações de objetos, tais como mapas e cartas. No que diz respeito às aplicações da cartografia, e com destaque para os mapas, Alvares (2019, p. 46), os toma como recursos essenciais "no planejamento, no estímulo ao raciocínio e na garantia da memória cultural, científica e tecnológica de grupos sociais". Assim, mais do que guias, mapas são resultados das relações de poder e, por sua vez, também instauram novas relações dessa natureza. Cada mapa, sentencia Lucy Fellowes, curadora da exposição The power of maps do CooperHewitt National Museum of Design (apud ALVARES, 2019, p. 46), "é a maneira de conseguir que o outro veja o mundo a sua maneira". As geotecnologias, como 
afirma Franco (2019, p. 90) "reafirmam a cartografia e o mapeamento como um meio de projetar poder e saber".

Nesse sentido, os produtos da cartografia tornam-se instrumentos importantes de visibilidade, de conquista de espaços e de demarcação de "territórios", tomando-se esse termo em seu sentido não literal e metafórico, naturalmente, pois no ciberespaço que caracteriza a web, isto é "o espaço de comunicação aberto pela interconexão mundial dos computadores e das memórias dos computadores" (LÉVY, 1999, p. 92), opera-se melhor com o conceito de desterritorialização (LÉVY, 1996).

O termo ciberespaço tem origem do grego kyber, que significa "para navegar" e é compreendido, de forma literal, como um "espaço navegável". Identifica-se comumente como a fonte original do termo, a fiç̧ão científica “Neuromancer", publicada pelo escritor estadunidense William Gibson em 1984. O ciberespaço constitui-se de modo não homogêneo, pois não se trata de um lugar ou de um bloco inconsútil, mas de uma quantidade indeterminada de ciberespaços em rápida expansão, viabilizados pelas tecnologias associadas à internet, à realidade virtual e às telecomunicações. O ciberespaço é considerado um meio poderoso para interações, incluindo-se cada vez mais, com o avanço das tecnologias digitais, as síncronas (DODGE; KITCHIN, 2001).

Paralelamente às características de pós-modernidade associadas ao ciberespaço, ou previamente a elas, as relações na sociedade moderna estabelecem-se em caráter de referencialidade - ou de georreferencialidade, para ser mais exato - às questões espaciais. Em referência à dicotomia contemporânea "espaço/falta de espaço" Dodge e Kitchin (2001, p. 13, tradução livre) apontam a ocupação política dos espaços:

[a]s pessoas e seus locais de produção e consumo são organizados em relação a uma lógica espacial ditada por fatores como o atrito da distância. Assim, as cidades se desenvolveram para superar o tempo com o espaço e se localizaram onde pudessem usufruir de matérias-primas ou rotas de comércio; lugares de produção localizados em relação a materiais e mercados para minimizar custos e maximizar vendas; e lugares de consumo localizados em lugares que devem maximizar vendas.

O mesmo ciberespaço que cria espaços públicos é, ao mesmo tempo, de modo quase paradoxal, um dos principais meios de monitoramento do espaço 
público no domínio geográfico (DODGE; KITCHIN, 2001). Entre outros fatores, isso se deve ao emprego das tecnologias de hiper-realidade como elementos que medeiam o espaço virtual e o espaço real, não sendo incomum que a representação do espaço real possa ser percebida como mais autêntica que o próprio real, isto é, atestando de modo explícito a cultura do simulacro como apontada por Baudrillard (1983). Nesse sentido, tem se popularizado a organização e representação de informações sobre lugares e pessoas que se Ihes relacionam com o recurso de ambientes geográficos virtuais.

O virtual, que não deve ser confundido com o "possível", bem como seus efeitos, vale recordar a advertência de Lévy (1996), não se opõe ao real, mas ao atual. Assim, a virtualização

não é uma desrealização (a transformação de uma realidade num conjunto de possíveis), mas uma mutação de identidade, um deslocamento do centro de gravidade ontológico do objeto considerado: em vez de se definir principalmente por sua atualidade (uma "solução"), a entidade passa a encontrar sua consistência essencial num campo problemático [...] (LÉVY, 1996, p. 17-18).

A diferença entre o possível e o real, portanto, é compreendida no domínio da lógica, pois o possível "é exatamente como o real: só lhe falta a existência (LÉVY, 1996, p. 16).

A invisibilidade do ciberespaço, portanto, não condiz com a crença em uma infraestrutura etérea e virtual, tais como a informação e a comunicação que ele suporta. Ao contrário, essa infraestrutura tem uma materialidade que pode ser mapeada no espaço geográfico e exibida por meio de técnicas cartográficas (DODGE; KITCHIN, 2001).

Considerando-se, portanto, o "deslocamento do centro de gravidade ontológico" (LÉVY, 1996), pode-se afirmar que o ciberespaço também possui formas arquitetônicas, ocorre que essas são desmaterializadas e dinâmicas, ou seja, são:

[...] espaços que não são fisicamente tangíveis, no sentido de que só podem ser explorados pela mente, mas metaforicamente relacionados com experiência corporal. Muitos espaços não têm contrapartes geográficas tangíveis - eles são espacializações utilizando uma metáfora geográfica para ganhar tangibilidade (DODGE; KITCHIN, 2001, p. 30, tradução livre).

A visualização geográfica, enquanto resultado do mapeamento 
cartográfico no ciberespaço, torna-se um desafio, pois confronta dois princípios considerados tradicionais da cartografia ocidental, que são: 1) o espaço é contínuo e ordenado; 2) o mapa não é o território, mas sim sua representação (DODGE; KITCHIN, 2001). Nos espaços de informação, destacam os mesmos autores no mesmo texto, a metáfora geográfica e o território tornam-se sinônimos, uma vez que o território se torna negociado em função da estruturação dos dados para o uso das espacializações. A linguagem para modelagem em realidade virtual (VRML, na sigla em inglês) possibilita às pessoas a visualização e a navegação nos espaços geográficos, tais como aquelas que são empregadas nos diversos instrumentos de localização e deslocamento disponíveis atualmente em veículos e telefones celulares, entre outros.

Dodge e Kitchin (2001) apontam três marcos evolutivos da cartografia no final do século XX: a) a popularização e o barateamento dos processos de digitalização, a disseminação da utilização de sistemas computadorizados, tais como os Sistemas de Informação Geográfica, mais conhecidos pela sigla em inglês "GIS", e o Design Auxiliado por Computador (CAD, na sigla em inglês); b) a criação de novos métodos de visualização geográfica; c) uma mudança de atitude sobre como os mapas são conceituados. O maior acesso à tecnologia de mapeamento e visualizações geográficas, estendem o poder do mapeamento de formas qualitativas e quantitativas.

Acrescenta-se a esse conjunto de tecnologias as facilidades de acesso promovidas por instrumentos populares e gratuitos como o Google Maps (no Brasil, disponível em: http://www.google.com.br), serviço lançado em 2005 pela empresa estadunidense Google, com amplo poder de alcance e cobertura geográfica. O Google Maps disponibiliza ferramentas de pesquisa, visualização e criação de mapas, com modos de visualização que incluem imagens de satélite. Tendo-se em vista sua gratuidade, popularidade e facilidade de uso, além do atendimento aos requisitos técnicos básicos de cartografia, essa ferramenta foi selecionada para a construção do mapa objeto desta pesquisa. 


\section{PROCEDIMENTOS METODOLÓGICOS}

A pesquisa foi desenvolvida em três fases. Na fase 1, foi realizado um levantamento junto aos programas de pós-graduação em ciência da informação avaliados e reconhecidos pelo Conselho Nacional da Educação (CNE/MEC) por meio da Plataforma Sucupira (disponível em: https://sucupira.capes.gov.br/ sucupira/). Na Plataforma Sucupira encontram-se, com acesso livre, dados sobre a Avaliação do Sistema Nacional de Pós-Graduação. Por meio desse recurso, foram recuperadas informações sobre dezoito programas de pós-graduação em ciência da informação no Brasil, assim distribuídos: Norte $=1$; Nordeste $=6$; $=$ Sul $=3 ;$ Sudeste $=7 ;$ Centro-Oeste $=1$. Realizou-se análise nas páginas web de cada uma das instituições listadas com a finalidade de identificar quais delas elencavam linhas de pesquisa sobre "organização do conhecimento", incluindo, por correlação semântica, "organização da informação" e "organização e representação do conhecimento". Foram identificadas treze linhas de pesquisa em nove instituições e enumerados na base de dados em construção todos os docentes inseridos em cada uma delas.

$\mathrm{Na}$ fase 2, utilizou-se a Plataforma Lattes para analisar o currículo Lattes dos docentes. A Plataforma Lattes, mantida pelo Conselho Nacional de Desenvolvimento Científico e Tecnológico (CNPq) possibilita ao pesquisador inserir informações curriculares sobre a atuação profissional e a produção acadêmica e científica de pesquisadores. Para os fins da coleta de dados, estabeleceu-se como filtro o preenchimento pelo docente do campo "linhas de pesquisas" envolvendo as temáticas: "organização do conhecimento", "organização da informação" e "organização e representação do conhecimento". A aplicação do filtro de pesquisa foi necessária porque quando foram analisadas as páginas web das instituições listadas identificaram-se informações discrepantes daquelas fornecidas no Lattes. Subsequentemente, descreveu-se o perfil dos pesquisadores, coletando-se as seguintes informações: nome, vínculo atual (universidade e departamento), endereço (URL) da instituição, URL do Lattes e foto de perfil (a que estava disponível no Lattes ou, na sua ausência, a que aparecia na página web da instituição). 
Para a consecução da fase 3 , foram utilizados os seguintes serviços do pacote Google: Planilha e Maps, com personalização pelo My Maps. A Planilha Google foi utilizada para automatizar o trabalho, cadastrando os perfis dos docentes para posteriormente importá-los no Google Maps, inclusive os dados de coordenadas geográficas. Todas as ferramentas são gratuitas e permitem, de modo relativamente simples, o compartilhamento de informações. Desse modo, criou-se um mapa denominado "pesquisadOCs" ao qual foram adicionadas camadas com as denominações das instituições recuperadas e subsequentemente, foram importados os perfis dos docentes listados na Planilha Google, aninhando-se tais perfis nas camadas do mapa.

\section{A CONSTRUÇÃO DO MAPA DA ORGANIZAÇÃO DO CONHECIMENTO NO BRASIL}

Alguns aspectos do mapa produzido para ilustrar os resultados nesta pesquisa estão representados nas Figuras 2, 3 E 4 . O mapa completo, com suas interações, pode ser acessado em: https://bit.ly/2UOqmuV ou pelo QRCode da Figura 1.

Figura 1 - QRCode para acesso ao mapa da base pesquisadOCs

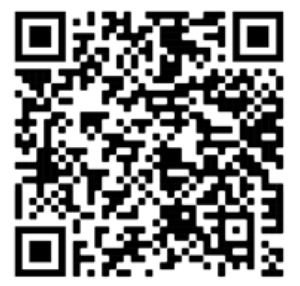

Fonte: Elaborado pelos autores (2021)

Os resultados apresentados ainda são iniciais, pois se trata de um programa piloto para um projeto em andamento que pretende ampliar a representatividade e a qualidade da cobertura nacional e incluir a comunidade internacional de pesquisadores do campo da organização do conhecimento vinculados à ISKO em seus diversos capítulos. Os diversos critérios e procedimentos adotados nesta pesquisa servem, portanto, também para a calibragem dos procedimentos metodológicos ampliados, isto é, para os ajustes necessários nos procedimentos e instrumentos de coleta de dados com vistas à 
sistematização de dados mais complexos conforme a ampliação dos territórios cobertos e a inclusão de outros elementos descritivos.

\section{Figura 2 - Mapa da base pesquisadOCs: visão geral}

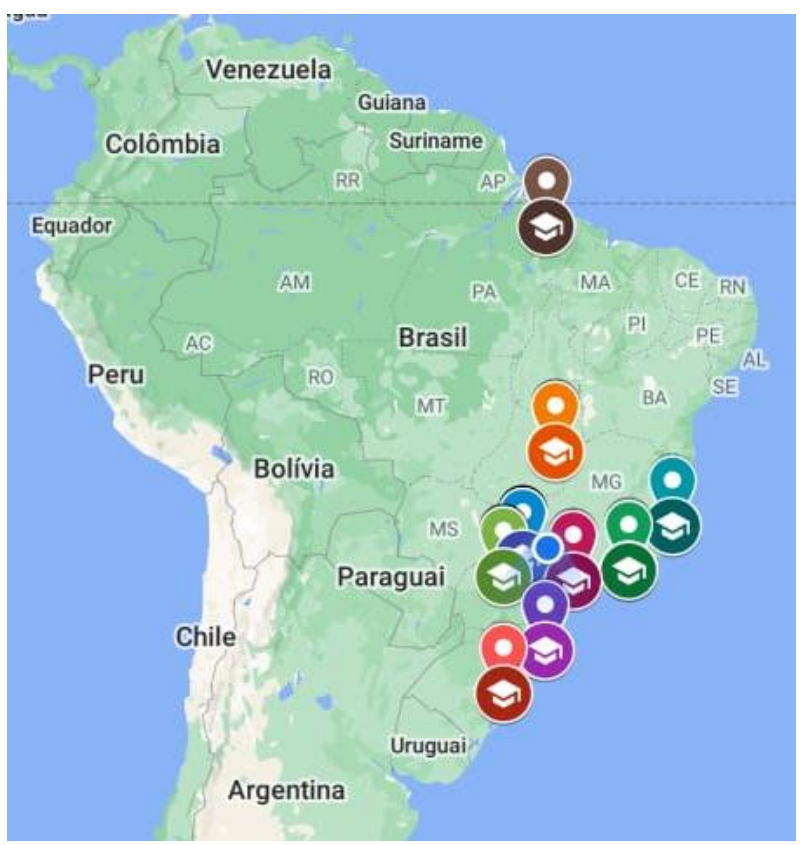

Fonte: Elaborado pelos autores (2021)

O conjunto de docentes identificados e que compõem a base estão distribuídos nas seguintes regiões e universidades: Norte $=1$ docente (UFPA); Nordeste $=0$; Centro-Oeste $=2$ docentes $(\mathrm{UnB}) ; \mathrm{Sul}=8$ docentes $(\mathrm{UEL}=5 ; \mathrm{UFRGS}=2 ; \mathrm{UFSC}=2$ ) e Sudeste $=13$ docentes $(\mathrm{UFES}=1 ; \mathrm{UFF}=1 ; \mathrm{UFRJ}=1 ; \mathrm{UNESP}=10$ ).

Figura 3 - Mapa da base pesquisadOCs: visão parcial por universidade

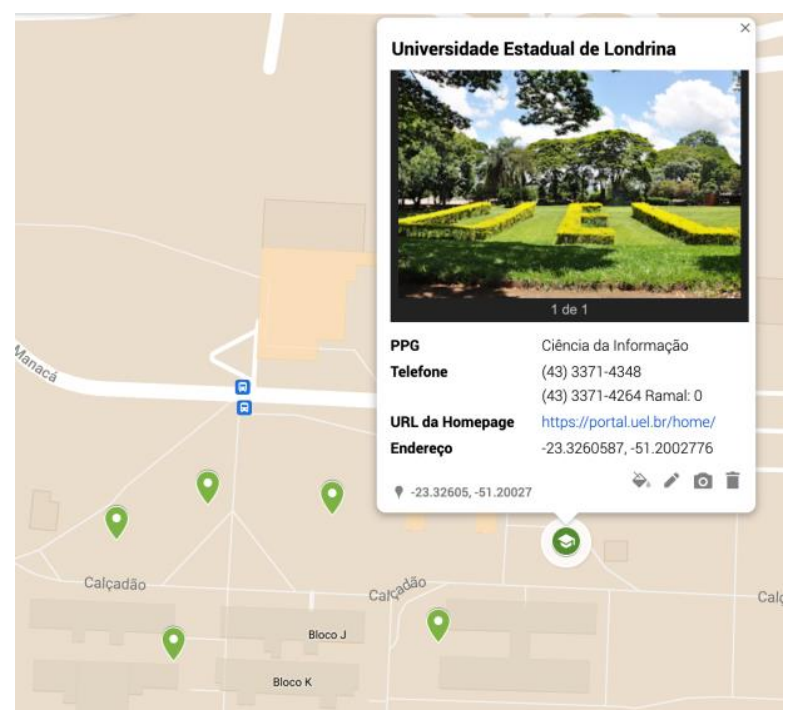

Fonte: Elaborado pelos autores (2021) 
O número total de docentes $(\mathrm{n}=24)$ não reflete, claro está, a totalidade da comunidade brasileira de pesquisadores, mas é, de qualquer modo, pode-se garantir, uma amostra representativa. Considerando-se que para a construção do mapa ateve-se apenas aos dados identificados conforme os critérios definidos nesta pesquisa, há, como se poderá notar pela consulta ao mapa, ausência de alguns outros pesquisadores com reconhecido destaque na pesquisa em organização do conhecimento desenvolvida no Brasil. Uma simples lista com recurso à memória já poderia incluir muitos outros, mas poderia deixar de inserir, igualmente, outros vários. Assim, optou-se pela fidelidade absoluta aos critérios da pesquisa, isto é, a inserção de dados apenas quando fosse observada a presença dos termos "organização do conhecimento" ou suas variações semânticas no campo "linhas de pesquisa" dos respectivos currículos Lattes. Em diversos casos, portanto, o pesquisador não figura listado simplesmente porque não havia preenchimento desse campo no seu currículo Lattes.

Figura 3 - Mapa da base pesquisadOCs: visão parcial por pesquisador

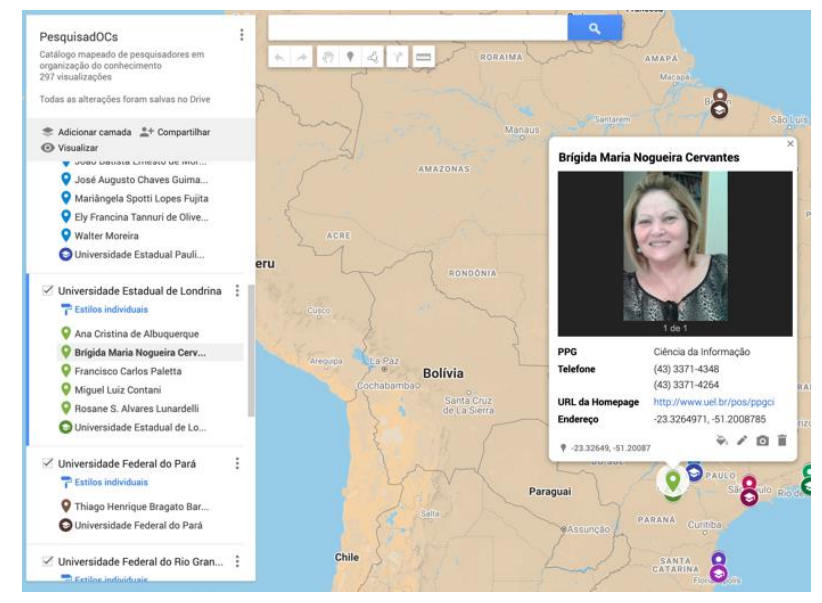

Fonte: Elaborado pelos autores (2021)

Considera-se que a pesquisa cumpriu os objetivos propostos e apresentou resultados bastante satisfatórios. Para a coleta de dados foram tomadas duas bases absolutamente validadas e com ampla representatividade institucional da comunidade mapeada, nomeadamente, a base nacional de currículos Lattes e a plataforma Sucupira. Isso permitiu identificar os centros de referência em pesquisas sobre organização do conhecimento no Brasil, todos 
eles vinculados a universidades públicas nas esferas federal e estadual.

\section{CONSIDERAÇÕES FINAIS}

O mapa apresentado possibilita localizar e identificar rapidamente endereços, contatos e outras informações breves de pesquisadores cujos temas centrais se relacionem à organização do conhecimento, facilitando eventuais contatos, inclusive com apresentação de coordenadas geográficas, o que pode ser utilizado para a exportação dos dados cadastrados para outras bases ou softwares de gestão da informação cartográfica mais robustos.

No desenvolvimento do projeto, pretende-se ampliar a base de dados por meio de definição de novos parâmetros e de nova análise de currículos Lattes de pesquisadores brasileiros. Na sequência, pretende-se construir o mapa da comunidade internacional, incluindo a comunidade de pesquisadores internacionais vinculados à ISKO.

\section{REFERÊNCIAS}

ALVARES, L. M. A. R. Cartografia de bibliotecas em ciência e tecnologia no Brasil: análise espacial como estratégia para a construção de políticas de informação científica e tecnológica. Informação \& Sociedade, Brasília, v. 13, n. 1, p. 44-54, jul./dez. 2019.

BARITÉ, M. Organización del conocimiento: un nuevo marco teórico-conceptual en bibliotecología y documentación. In: CARRARA, K. (org.). Educação, universidade e pesquisa. Marília: UNESP-Marília, 2001. p. 35-60.

BAUDRILLARD, J. Simulacra and Simulations. New York: Semiotext(e), 1983.

DODGE, M.; KITCHIN, R. Mapping cyberspace. London: Routledge, 2001.

FRANCO, J. R. Cartografias criativas: da razão cartográfica às mídias móveis. Curitiba: Appris, 2019.

GUIMARÃES, J. A. C. A análise documentária no âmbito do tratamento da informação: elementos históricos e conceituais. In: RODRIGUES, G. M.; LOPES, I. L. (org.) Organização e representação do conhecimento na perspectiva da ciência da informação. Brasília: Thesaurus, 2003. p. 100-117. 
GUIMARÃES, J. A. C. A Organização do conhecimento: passado, presente e futuro sob a perspectiva da ISKO. Informação \& Informação, Londrina, v. 22, n. 2, p. 84-98, 2017.

LÉVY, P. O que é o virtual? São Paulo: 34, 1996.

LÉVY, P. Cibercultura. São Paulo: 34, 1999.

MOREIRA, W. Os colégios virtuais e a nova configuração da comunicação científica. Ciência da Informação, Brasília, v. 34, n. 1, p. 57-63, 2005.

OLIVEIRA, C. Dicionário cartográfico. 4. ed. Rio de Janeiro: IBGE, 1993.

\title{
“PESQUISADOCs": ELEMENTS FOR THE CARTOGRAPHY OF THE KNOWLEDGE ORGANIZATION BRAZILIAN COMMUNITY OF RESEARCHERS
}

\begin{abstract}
Objective: Considering the volume and the geographic dispersion of the community of researchers who study the theme of knowledge organization in Brazil, the objective is to identify, describe and map, with the aid of geographic coordinates, the reference centers and researchers involved with Brazilian research on knowledge organization. Methodology: These researchers, linked to information science postgraduate programs in Brazil, were identified through their respective Lattes curricula and, additionally, through the collection of information from the web pages of the programs in which they work. Google Maps was used as a technological resource for data collection and for the construction and availability of cartographic information. Results: In this first approach, the map produced identifies and catalogs a set of 24 researchers distributed in 9 institutions covering the entire Brazilian territory. Conclusions: The presented map makes it possible to quickly locate and identify addresses, contacts, and other brief information of researchers whose central themes are related to the organization of knowledge, facilitating eventual contacts, including the presentation of geographic coordinates, which can be used for data export registered for other databases or more robust cartographic information management software.
\end{abstract}

Descriptors: Knowledge organization. Community of researchers. Cartography.

\section{“PESQUISADOCS": ELEMENTOS PARA LA CARTOGRAFÍA DE LA COMUNIDADE BRASILEÑA DE INVESTIGADORES EN ORGANIZACIÓN DEL CONOCIMIENTO}

\section{RESUMEN}

Objetivo: Considerando el tamaño y la dispersión geográfica de la comunidad de investigadores que estudian el tema de la organización del conocimiento, el objetivo es 
identificar, describir y mapear, con el recurso de las coordenadas geográficas, los centros de referencia e investigadores involucrados con la investigación brasileña sobre el conocimiento. organización. Metodología: Estos investigadores, vinculados a programas de posgrado en Brasil, fueron identificados a través de sus respectivos currículos Lattes y, adicionalmente, a través de la recolección de información de las páginas web de los programas en los que trabajan. Se utilizó Google Maps como recurso tecnológico para la recolección de datos y para la construcción y disponibilidad de información cartográfica. Resultados: En esta primera aproximación, el mapa elaborado identifica y cataloga un conjunto de 24 profesores distribuidos en 9 instituciones que cubren todo el territorio brasileño. Conclusiones: El mapa presentado permite ubicar e identificar rápidamente direcciones, contactos y otra información breve de investigadores cuyos temas centrales se relacionan con la organización del conocimiento, facilitando eventuales contactos, incluyendo la presentación de coordenadas geográficas, que pueden ser utilizadas para la exportación de datos registrados para otras bases de datos o software de gestión de información cartográfica más robusto.

Descriptores: Organización del conocimiento. Comunidad de investigadores. Cartografía.

Recebido em: 15.10 .2021

Aceito em: 27.12.2021 